\title{
Single-photon interference experiment for high schools
}

\section{Maria Bondani}

Maria Bondani, "Single-photon interference experiment for high schools," Proc. SPIE 9289, 12th Education and Training in Optics and Photonics Conference, $92890 \mathrm{H}$ (17 July 2014); doi: 10.1117/12.2070772

SPIE Event: 12th Education and Training in Optics and Photonics Conference, SPIE. 2013, Porto, Portugal 


\title{
Single-photon interference experiment for High Schools
}

\author{
Maria Bondani \\ Institute for Photonics and Nanotechnologies, CNR; CNISM UdR Como; \\ Dipartimento di Scienza e Alta Tecnologia, Università degli Studi dell'Insubria, \\ via Valleggio 11, 22100 Como, Italy
}

\begin{abstract}
We follow the reductio ad absurdum reasoning described in the book "Sneaking a Look at God's Cards" by Giancarlo Ghirardi to demonstrate the wave-particle duality of light in a Mach-Zehnder interferometric setup analog to the conventional Young double-slit experiment. We aim at showing the double nature of light by measuring the existence of interference fringes down to the single-photon level. The setup includes a strongly attenuated laser, polarizing beam splitters, half-waveplates, polarizers and single-photon detectors.
\end{abstract}

Keywords: High School, Quantum Mechanics, Young Interference, single-photon detectors

\section{INTRODUCTION}

Conventional presentations of the principles of Quantum Mechanics in High School programs follow a historical approach. In this context, a number of milestone experiments of the beginning of the XX Century are usually discussed: the problem of blackbody radiation, the interpretation of the photoelectric effect, the structure of atom and the interpretation of emission and absorption spectra, the diffraction of electrons. Schrödinger equation and the interpretation of wavefunctions are rarely introduced, even at the elementary level: for this reason the superposition principle, which is the origin of the weirdness of Quantum Mechanics, remains unexplained.

In particular, what is not pointed out is that Schrödinger equation is linear and that, therefore, if $\psi_{1}$ and $\psi_{2}$ are two solutions, the function $\psi_{1}+\psi_{2}$ is also solution. It follows that if a quantum object admits two states described by the wavefunctions $\psi_{1}$ and $\psi_{2}$, this object can also be described by a wavefunction that is a linear combination of the type $\psi=a \psi_{1}+b \psi_{2}$ : this is a superposition state.

The most important consequence of this results is in the difference between the concept of probability according to Classical Physics and according to Quantum Physics. In the first case, a system is in the state $\psi_{1}$ OR in the state $\psi_{2}$, and therefore the probability of finding the system in the state $\psi_{12}$ is given by the sum of the probabilities of the two states according to the relation $p_{12}=\left|a \psi_{1}\right|^{2}+\left|b \psi_{2}\right|^{2}$. In the second case, the system is in the state $\psi_{1}$ AND in the state $\psi_{2}$ as the two states are not mutually exclusive but can be considered simultaneously present, so the probability of finding the system in one of the two states is given by $p_{12}=\left|a \psi_{1}+b \psi_{2}\right|^{2}=\left|a \psi_{1}\right|^{2}+\left|b \psi_{2}\right|^{2}+2\left|a \psi_{1}\right|\left|b \psi_{2}\right| \cos (\phi)$, where $\phi$ is the phase difference between wavefunctions $\psi_{1}$ and $\psi_{2}$. This specific feature of the theory is essential to understand Quantum Mechanics.

On the other hand, to illustrate the existence of superposition states, textbooks often describe single-electron interference ${ }^{1}$ that takes place when electrons impinge on a double slit one at a time. Unfortunately this experiment requires a high-level equipment that prevents it from being easily reproduced.

Here we present a different experiment in which the superposition of states for polarized single photons is discussed. The possible states considered are the two different paths inside a Mach-Zehnder interferometer ${ }^{2,3}$ equipped with two polarizing beam-splitters, so that the paths identify a given polarization.

Several setups have been proposed to study single-photon quantum effects by producing photon pairs by parametric down-conversion in nonlinear crystals. ${ }^{4-6}$ The required apparatus is definitely beyond the possibilities of Secondary Schools and, even if it can be built in University laboratories, it can hardly be transported in schools. For this reason we decided to work on an approximation of the single-photon state obtained by attenuating a laser beam down to the so-called "single-photon" level, that is a condition in which single-photon avalanche detectors click very rarely. ${ }^{7}$ Strictly speaking, this is not a single-photon state, since the photon-number statistics of the state is poissonian and we always have some probability to have more than one photon in a single avalanche of the detector. Nevertheless the error can be reduced by reducing the mean value of the detection rate.

Further author information: (Send correspondence to M.B.: E-mail: maria.bondani@uninsubria.it) 


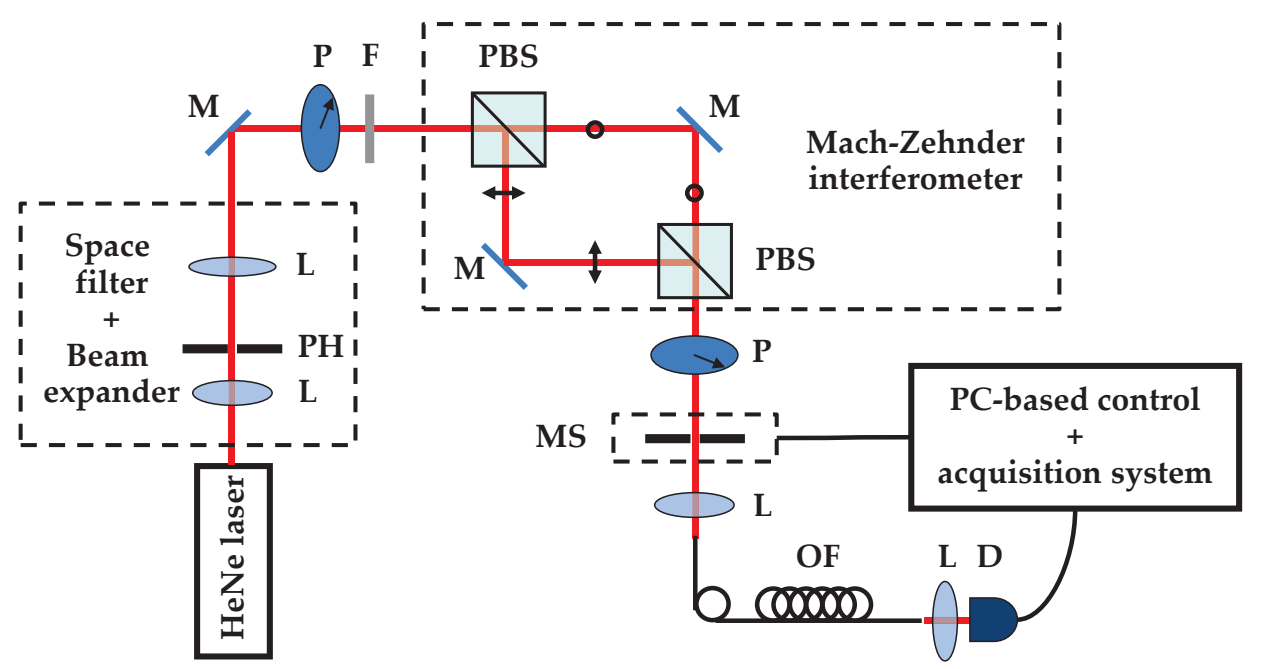

Figure 1. Scheme of the experimental setup.

\section{DIDACTIC ROUTE}

The peculiarity of the experiment requires a specific training for both teachers and students. Several weeks before the execution of the activity with their classes, teachers are invited to attend two lectures during which the basics of Quantum Mechanics are discussed with particular attention to superposition states, to interpretation of wavefunctions and to meaning of probability and, in the second lecture, the experiment is described in detail. Teachers are then supposed to organize lectures for their classes to introduce the topic of single-photon interference. An introduction to the logic of the experiment will be delivered to students the same day of the experiment execution.

The experimental apparatus is depicted in Fig. 1. A spatially filtered and enlarged laser beam is strongly
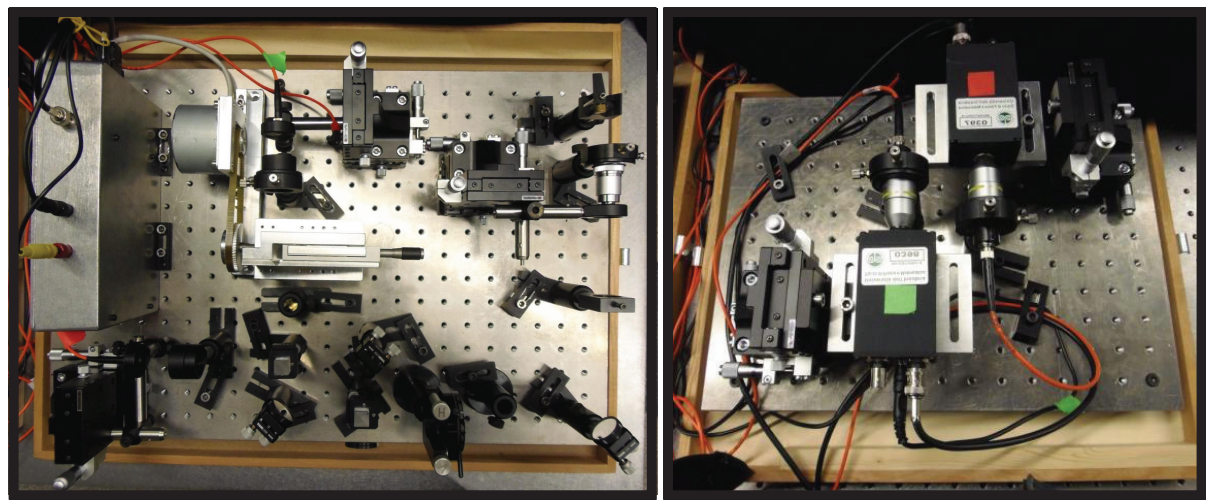

Figure 2. Portable experimental apparatus: optical setup and detectors are mounted on two separate breadboards and the light is delivered to detectors through optical fibers.

attenuated, polarized by a sheet polarizer $(\mathrm{P})$ and injected into a Mach-Zehnder interferometer realized with two polarizing beam splitters (PBS). A second sheet polarizer is placed at the output of the interferometer and the light passing through it is finally collected by a lens (an achromatic doublet) and focused into an optical fiber that delivers it to a single-photon avalanche detector (SPAD, MPD, Bolzano, Italy, > 50\% detection efficiency). The output signal of the detector is finally processed by a counter and stored in a computer.

The experiment follows step-by-step the reasoning described in Ghirardi's book. ${ }^{8}$ It is a reductio ad absurdum argument that leads the reader through a series of apparently solid facts and finally reaches an unexpected logical contradiction. 
In the following we list the steps of the logical path.

\section{Step 0}

We assume that light is composed by indivisible particles called photons and we demonstrate that the detectors can measure single photons: this is essential for the following consideration. We lower the light well beyond visibility and count the single detector events (clicks) in given time intervals. The clicks are randomly distributed and the count statistics is Poissonian.

\section{Step 1}

We demonstrate that the polarizing beam splitters divide the light according to polarization even in the single photon regime. If the impinging photons are vertically or horizontally polarized, they will either be transmitted or reflected (see Fig. 3(a)) and (b) with unit probability. The result is not surprising because the initial state of photons is aligned to one of the axes of the polarizer. Then we demonstrate that $45^{\circ}$-polarized single photons are never measured at the two outputs of a beam splitter simultaneously by verifying that two single-photon detectors at the two outputs never "click" together, but they register light half of the time each. We can thus conclude that photons at the bean splitter behave like indivisible particles that randomly exit at one of the outputs so that, on the average, the light is divided in two equal parts Fig. 3)(c). This leads us to the opinion that photons follow a defined path inside the interferometer.

(a)

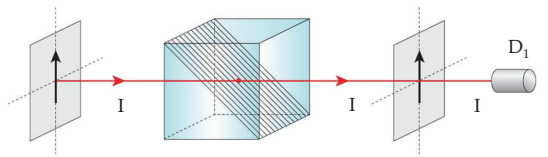

(b)

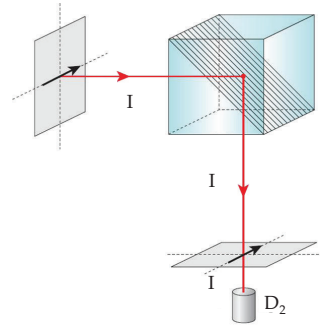

(c)

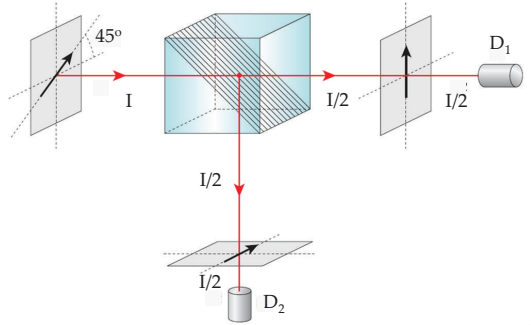

Figure 3. Behavior of single photons passing through a polarizing beam splitter: if the initial polarization is parallel to one of the optical axes, the light is transmitted $(a)$ or reflected $(b)$, if polarization is at $45^{\circ}$, half of the light is transmitted and half is reflected $(c)$.

\section{Step 2}

We inject $45^{\circ}$-polarized single photons into the interferometer. If the initial intensity (that is the mean number of detector counts registered by the detector) is $I$, the intensity measured through a vertical or horizontal polarizer by stopping one of the paths is $I / 2$ on each arm (see Fig.4(a)), like for macroscopic light. The fraction of detected clicks sets the probability of finding the photon in each of the two arms. On the other hand, if the final polarizer is rotated to $45^{\circ}$, the light intensity measured by stopping the paths one at a time is $I / 4$ (see Fig.4(b)): also polarizers act on single photons by either stopping or transmitting them but never dividing photons in two parts, and the number of counts gives the probability that the photon passes the polarizer. Again the behavior at the single-photon level reproduces the macroscopic behavior on the average.

At this point of the reasoning we should be convinced that one $45^{\circ}$-polarized single photon entering the interferometer would randomly choose one of the two paths inside the interferometer and that only one half of the photons in each arm would pass the final $45^{\circ}$-oriented polarizer. 
(a)

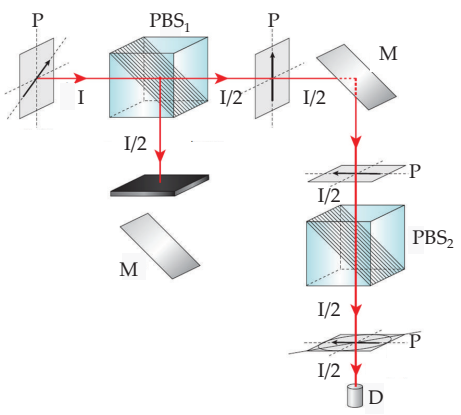

(b)

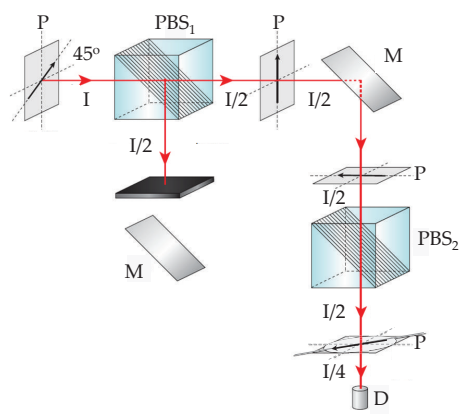

Figure 4. Behavior of single photons travelling through one arm of the interferometer when the other arm is stopped. The mean value of detected photons depends on the orientation of the optical axis of the final polarizer: $(a)$ for parallel orientation, the transmitted light is $I / 2 ;(b)$ for orientation at $45^{\circ}$, the transmitted light is $I / 4$.

\section{Step 3}

Now we open both arms of the interferometer and measure the output intensity through a $45^{\circ}$-polarizer. According to Step 2, the expected number of photons should be the sum of those following the two paths in the Mach-Zehnder, that is $I / 4+I / 4=I / 2$. What we instead observe is that the measured mean number of photons can vary between $I$ and 0 as a function of the mirror position: this result says that the total probability of detecting a photon at the output of the interferometer is not the sum of the probabilities that the photon follow the two paths inside of it. The final observation is thus incompatible with the previous conclusions that assigned to photons particle-like characteristics supported by the observation that they seem to follow a well defined path inside the interferometer and closes the reductio ad absurdum reasoning by showing the inconsistence of the hypothesis that photons are indivisible particles. The only way to understand the result is to assume a wave-like behavior of the photons that would allow them to follow both paths in the interferometer at the same time, giving rise to interference. Note that for polarizer axis parallel to one of the axes of the PBS the measured intensity is $I / 2$ like in Step 2: we obtain interference fringes only for the final polarizer with the same orientation parallel to the input-photon polarization.

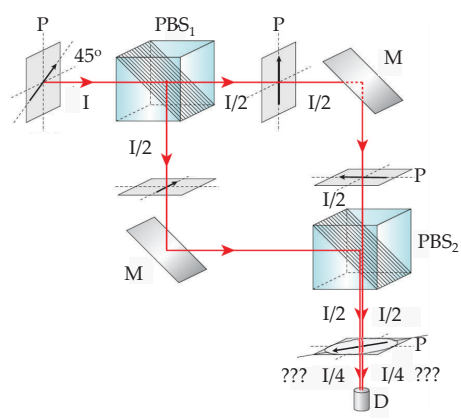

Figure 5. Behavior of single photons travelling through both arms of the interferometer: the final detected number of photons depends on the mirrors' alignment, like in interferometry.

\section{Step 4}

Since the behavior described in Step 3 is that of an interference, we can scan the interference pattern to demonstrate it over a large number of positions. We do this by scanning the interference fringes at single-photon level by collecting the light through a narrow slit that moves along the pattern. In Fig. $6(a)$ the schematic of the system is described. In Fig. 6(b) the result of the scanning is presented: we acquired a full scan of the interference 
$(I(x))$ and also full scans of the single beams $\left(I_{1}(x)\right.$ and $\left.I_{2}(x)\right)$ in order to calculate the quantity $\cos (\phi)$ according to the formula:

$$
I(x)=I_{1}(x)+I_{2}(x)+2 \sqrt{I_{1}(x) I_{2}(x)} \cos (\phi) .
$$

The values of $\cos (\phi)$ are plotted in the lower part of Fig. 6(b).

At the end of the experiment we have two incompatible sets of observations: the first one says that photons

(a)

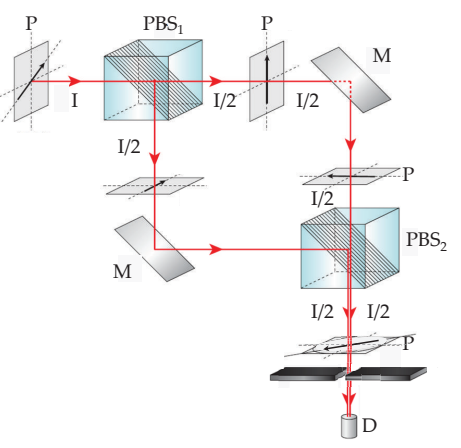

(b)

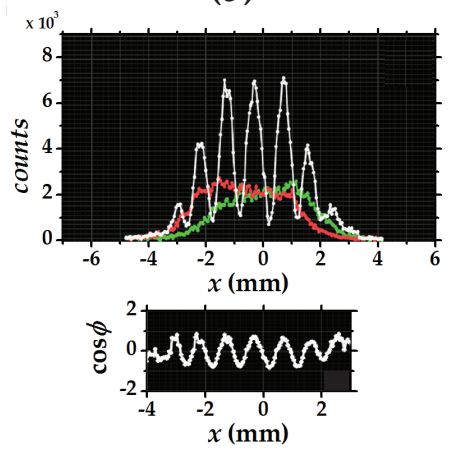

Figure 6. (a): Scheme for the measurement of the interference fringes at single-photon level by scanning the detector through the fringe pattern and accumulating the counts; $(b)$ : experimental results for the interference pattern (upper panel) and experimental values of $\cos (\phi)$ (lower panel).

never divide in parts and when measured by single-photon detectors behave like particles; the second one says that to account for the final result we have to consider photons as waves. This wave-particle duality, or in other words, the application of the superposition principle to the state of the photons inside the interferometer is the explanation of the experimental observations.

\section{DIDACTIC IMPLEMENTATION AND FOLLOW UP}

The overall activity with students requires about three hours, including introduction. Time is necessary to proceed very slowly along the logical reasoning avoiding errors. The topic is rather difficult and the reasoning unusual, more similar to philosophy than to experimental physics, but part of the appeal of the subject is in its oddity.

The activity was initially proposed in 2006 as a laboratory course for University students (Prof. Fabio Ferri). Together with students we set-up the first version of the experimental apparatus in the laboratories of the Department of Science and High Technology of University of Insubria at Como. At the beginning of 2007 we started involving High School classes in the framework of the "Progetto Lauree Scientifiche" of the Italian Ministry of Education. Classes had to come to University to perform the experiment. In 2009 we realized a portable version of the apparatus, reasonably stable and easy to align, to bring the experiment directly in the High Schools. Till now the didactic activity was performed with more than twenty classes, mainly from scientifically oriented High Schools. We decided to involve the entire class group, so that the activity was included in the regular Physics program and the class teacher. This choice brings together students having different levels of mathematical and physical skill and different levels of interest in the problem. The feedback of students about the activity is thus non uniform, even if positive on the average, and strongly depends on the level of the personal involvement with the activity the teachers had. In fact, not surprisingly, best results and appreciation came from students whose teachers actively participated in the preparation of the activity, by attending the introductory lectures at University and by developing suitable lectures for their students. The level of students' preparation could be appreciated from the questions they arose during the experimental activity more than from the correct answers they gave. In fact, as the topic is hard to handle even for specialists, being able to pose questions is the signature of the beginning of understanding.

The collaboration with the classes and their teachers led to the production of certain number of works presented at the High School licence exam. Moreover, the activity was presented to some national workshops ${ }^{9,10}$ and some 
teachers participating in the activity wrote educational papers describing the topic and the development of the activity. ${ }^{11,12}$

As a final remark, we note that the experimental setup is rather complicate and includes a lot of optical devices whose role in the experiment is shortly explained to students. A complete description of each part of the apparatus (spatial filter, polarizer, optical fiber, detectors...) is far beyond the possibilities of this activity but could be the subject of some expansion module of students' optics curricula.

\section{ACKNOWLEDGMENTS}

The Author wants to thank Fabio Ferri and Davide Magatti (Insubria), for participating to the initial setting of the experiment and for helping to solve many technical problems.

\section{REFERENCES}

[1] http://www.bo.imm.cnr.it/users/lulli/downintel/electroninterfea.html

[2] Schneider, M. B. and LaPuma, I. A. "A simple experiment for discussion of quantum interference and which-way measurement," Am. J. Phys. 70(3), 266-271 (2002).

[3] Lukishova, S. G., Stroud, C. R. Jr., Bissell, L. Zimmerman, B. and Knox, W.H., "Teaching Experiments on Photon Quantum Mechanics," in Frontiers in Optics 2008/Laser Science XXIV/Plasmonics and Metamaterials/Optical Fabrication and Testing, OSA Technical Digest (CD) (Optical Society of America, 2008), paper SThD3.

[4] Dehlinger, D. and Mitchell, M. W., "Entangled photon apparatus for the undergraduate laboratory," Am. J. Phys. 70(9), 898-902 (2002).

[5] Dehlinger, D. and Mitchell, M. W., "Entangled photons, nonlocality, and Bell inequalities in the undergraduate laboratory," Am. J. Phys. 70(9), 903-910 (2002).

[6] Pearson, B. J. and Jackson, D. P., "A hands-on introduction to single photons and quantum mechanics for undergraduates," Am. J. Phys. 78(5), 471-484 (2010).

[7] Marazzini P. and Bondani, M., "Insegnamento della meccanica quantistica nella scuola superiore. L'esperimento con fotoni polarizzati," http://gallery.pianetascuola.it/albums/galleria/risorse/ secondaria_secondo/fisica/marazzini_fotoni/Fotoni_polarizzati.pdf. In italian.

[8] Ghirardi, G. C., "Sneaking a Look at God's Cards, Revised Edition: Unraveling the Mysteries of Quantum Mechanics," (translated by Gerald Malsbary, Princeton University Press, Princeton, New Jersey USA, 2007). Original edition in italian: G. C. Ghirardi, "Un'occhiata alle carte di Dio" (Il Saggiatore, Milano, Italy, 2001).

[9] Liceo Scientifico "G. Ferraris" (Varese), Workshop "Ricerco anch'io: la parola agli studenti" - Joint Research Centre ISPRA (VA), $16 / 11 / 2006$.

[10] Meeting Progetto Lauree Scientifiche, Catania, 12/7/2007.

[11] Pavan, M., "Interferenza di singoli fotoni," Emmeciquadro 31, 142-151 (2007). In italian.

[12] Iotti, P., "Il fotone sa sempre dove andare?," Emmeciquadro. In preparation. 